\title{
Multi-photon Time-lapse Microscopy and Optical Recording to Study Neural Processing and Plasticity
}

\author{
Steve M. Potter ${ }^{1}$, Thomas B. DeMarse ${ }^{1}$, Axel W. Blau ${ }^{2}$, Daniel A. Wagenaar ${ }^{3}$ \\ 1*Laboratory for NeuroEngineering, Georgia Institute of Technology \& Emory School of Medicine, \\ Department of Biomedical Engineering, 315 Ferst Dr. NW, Atlanta, GA 30332-0535 \\ http://neuro.gatech.edu/potter \\ ${ }^{2}$ Dept. of Biophysics, University of Kaiserslautern, Germany \\ ${ }^{3}$ Dept. of Physics, California Institute of Technology
}

It has long been assumed that the learning process changes the shape and connectivity of the cells in our brains. But no one has been able to observe this process while it is happening. By combining advanced microscopic imaging techniques with multi-electrode array recording and stimulation, we can determine the relationships between the morphology of brain cells and their electrical activity. We developed a system for studying learning and memory in cultured neuronal networks (Fig.1). By using in vitro approaches, one has access to every cell in the neural circuit, something that is not possible in living animals.

One key element missing from previous in vitro approaches was behavior. A neural circuit growing in a dish is clearly missing the means to express behavior, and thus, to learn from experience. To address this fundamental deficiency of in vitro approaches, we have created a real-time two-way connection between cultured networks and a computer [1]. Dissociated monolayer cultures of rat cortex are grown on multi-electrode arrays (MultiChannel Systems) for up to two years [2]. On the computer is a simulated animal, the Neurally Controlled Animat, whose behavior is controlled by the neural activity recorded from 60 substrate electrodes. As the Animat behaves in its simulated environment, its sensory input is fed back to the neurons as patterns of electrical stimulation. We have also used the cultued nets as the 'brain' of a hybrid neural-robotic animal, or Hybrot. As the body, we used the Khepera wheeled robot (K-Team). Under neural control, the Hybrot was capable of tracking a light source. We are testing a variety of sensory-motor mapping schemes in the simulated Animats and the Hybrots, to be able to demonstrate learning and adaptation.

We have also developed a number of labeling and imaging techniques that allow us to do submicron resolution imaging, in three dimensions, across hours or days, without harming the cells being imaged. Long-term 4D imaging [3] of a neural circuits during learning is now possible, thanks to the greatly reduced photodamage of multi-photon laser-scanning microscopy, and to the durability of fluorescent proteins. We have observed changes in dendritic spines and neural connectivity in cultured nets that may provide insights into what changes about our brain cells when we learn something.

We built a high-speed CCD camera capable of optically recording spontaneous action potentials in single cultured mammalian neurons labeled with voltage-sensitive dyes. Data from optical recording is complementary to data from the substrate electrodes, e.g., to determine the spatial influence of an applied stimulus. The combination of optical and electrical recording and stimulation enables the study of distributed information processing in neuronal networks[4]. And by adding the 
morphometric capabilities of 2-photon microscopy, we can get at how the propagation of information through a network may induce lasting changes in its dynamics.

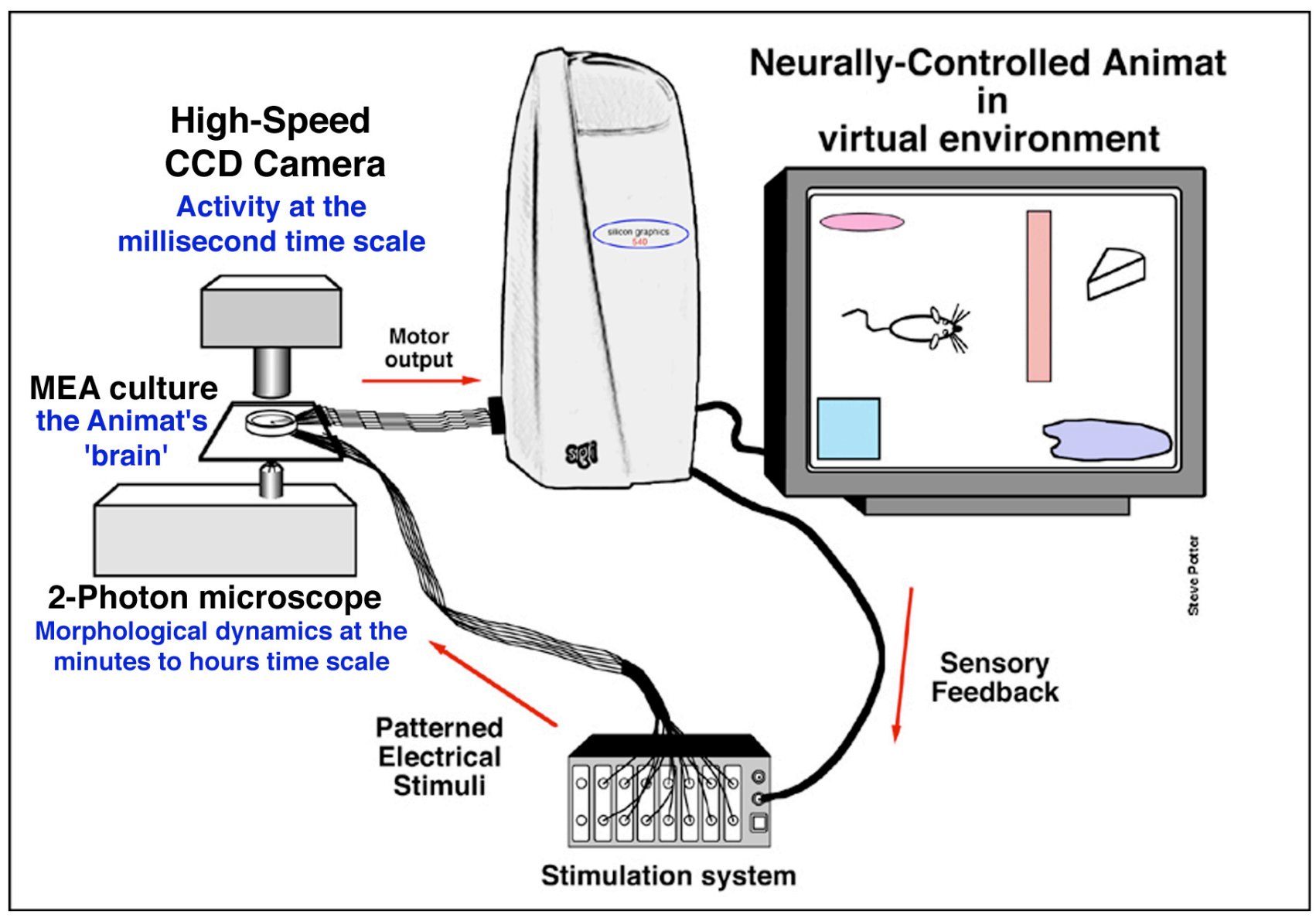

Fig. 1. System for studying learning and neural information processing in vitro. Neurons and glia are cultured in a monolayer network on multi-electrode arrays for months. These are transparent and amenable to submicron vital imaging using 2-photon time-lapse microscopy with fluorescent protein labels. Electrical signals produced by the neurons can be recorded both with substrate electrodes and optically, using voltage-sensitive dyes. Recorded activity is used to control a simulated animal, or Animat, and the Animat's sensory input is fed back to the network in real-time, as patterns of electrical stimulation.

\section{References}

[1]T. B. DeMarse, D. A. Wagenaar, A. W. Blau, and S. M. Potter, "The Neurally Controlled Animat: Biological Brains Acting with Simulated Bodies," Autonomous Robots, vol. 11, pp. 305-310, 2001.

[2]S. M. Potter and T. B. DeMarse, "A new approach to neural cell culture for long-term studies," $J$. Neurosci. Methods, vol. 110, pp. 17-24, 2001.

[3]S. M. Potter, "Two-Photon Microscopy for 4D Imaging of Living Neurons," in Imaging Neurons: A Laboratory Manual, R. Yuste, F. Lanni, and A. Konnerth, Eds. Cold Spring Harbor: CSHL Press, 2000, pp. 20.1-20.16.

[4]S. M. Potter, "Distributed processing in cultured neuronal networks," in Progress In Brain Research: Advances in Neural Population Coding, vol. 130, M. A. L. Nicolelis, Ed. Amsterdam: Elsevier, 2001, pp. 49-62.

[5] Suppoted by the NIH, NINDS, grant RO1 NS38628. 